EPJ Web of Conferences 60, 17007 (2013)

DOI: $10.1051 /$ epjconf $/ 20136017007$

(C) Owned by the authors, published by EDP Sciences, 2013

\title{
Search for Long-Lived Particles and Lepton-Jets with the ATLAS detector
}

\author{
D. Salvatore ${ }^{1, a}$ On behalf of the ATLAS Collaboration \\ ${ }^{1}$ University of Calabria, ponte P. Bucci, 87036 Rende (CS), Italy
}

\begin{abstract}
Many extensions of the Standard Model include neutral weakly-coupled particles that can be longlived. These long-lived particles occur in many models, included gauge-mediated extensions of the Minimal Supersymmetric Model (MSSM), MSSM with R-parity violation, inelastic dark matter and the Hidden Valley scenario. Results are presented on the ATLAS searches at the LHC for possible rare Higgs boson decays to pair of neutral, long-lived hidden-sector particles that lead to final states containing collimated lepton jets or fermion anti-fermion pairs. No excess of events above the expected background has been observed on data collected in 2011 at a center of mass energy of $7 \mathrm{TeV}$ and limits on the cross sections are set.
\end{abstract}

\section{Introduction}

Recently, the production of a boson with a mass of about $125 \mathrm{GeV}$ has been observed by the ATLAS [1] and CMS [2] Collaborations. The observation is compatible with the production and decay of the Standard Model (SM) Higgs boson at this mass. Strengthening or rejecting the SM Higgs boson hypothesis is currently of utmost importance and thus a search for non-SM Higgs boson decays is of high interest. Several models of physics beyond the SM predict the existence of Hidden Sectors able to comunicate to the SM through several portals (Higgs, Z', loop of SUSY particles). A light Higgs boson can decay to particles of the hidden sector [3] such as the long-lived pseudoscalar v-pion $\left(\pi_{v}\right)$ or scalar hidden fermions. These Hidden Valley (HV) particles can decay back in the standard sector to collimated jets of electrons or muons, lepton-jets (LJ) or fermion anti-fermion pairs. Lifetimes can be comparable to ATLAS [4] dimensions, leading to displaced decays far from the interaction point.

\section{Prompt lepton-jets}

This analysis [5] is based on a model where a pair of squarks is produced and each of the squarks cascade decays into dark-sector particles, including one or more dark-photons $\left(\gamma_{d}\right)$. The $\gamma_{d}^{\prime} s$ decay into pairs of leptons, forming LJs. Additionally, dark-sector particles may radiate multiple $\gamma_{d}^{\prime} s$, increasing the lepton multiplicities and number of the LJs. The amount of radiation is determined by the dark sector gauge coupling parameter $\alpha_{d}$. Setting $\alpha_{d}=0.0$ results in a simple LJ with two hard leptons. Larger values may produce LJs with four, six, eight, or more prompt leptons from the decay of overlapping $\gamma_{d}^{\prime} s$, albeit with reduced boost. The transverse momentum $\left(p_{\mathrm{T}}\right)$

\footnotetext{
ae-mail: daniela.salvatore@cern.ch
}

of the leptons increases with $\gamma_{d}$ mass, but decreases with $\alpha_{d}$. In this model, $\alpha_{d}=0.0,0.1$, and 0.3 , and $\gamma_{d}$ masses $\left(m_{\gamma}\right)$ of 150,300 , and $500 \mathrm{MeV}$ are used. For $m_{\gamma}=150$ $\mathrm{MeV}$, the $\gamma_{d}$ is below the $\mu^{+} \mu^{-}$threshold and can only decay to electrons. With $m_{\gamma} \geq 300 \mathrm{MeV}$, the $\gamma_{d}$ decays to electron and muon pairs. Additionally, for $m_{\gamma}=500 \mathrm{MeV}$, $20 \%$ of the decays produce pion pairs. This analysis considers LJs in three signatures: single muon-jets with four or more muons, pairs of muon-jets each with two or more muons, and pairs of electron-jets each with two or more electrons.

Events containing electron-jets (EJs) were selected using single-electron triggers with an online $p_{\mathrm{T}}$ threshold of 20 or $22 \mathrm{GeV}$. To ensure proper modelling of the trigger acceptance, events were required to contain at least one reconstructed electron with $p_{\mathrm{T}}>35 \mathrm{GeV}$, above which the trigger efficiency is constant. The reconstructed electron was required to match an electron reconstructed above the $p_{\mathrm{T}}$ threshold in the trigger system with a separation $\Delta R$ less than 0.2. The EJ candidates were built from electromagnetic (EM) clusters. At least two tracks from the primary vertex $(\mathrm{PV})$ with $p_{\mathrm{T}}>10 \mathrm{GeV}$ were required to have $\Delta R<0.1$ of the cluster position in the second sampling layer of the calorimeter. Additional requirements were made on the number of hits along the track in the silicon pixel and microstrip detectors to suppress backgrounds from photon conversions. The analysis required two LJ candidates in each event, with one cluster matching the electron reconstructed in the trigger system. The invariant mass of the two highest- $p_{\mathrm{T}}$ tracks associated with each EJ had to be less than $2 \mathrm{GeV}$. To reduce the background coming from multi-jet events, the electron cluster concentration $\left(R_{\eta 2}\right)$, defined as the ratio of total energy in $3 \times 7$ cells to the total energy in $7 \times 7$ cells in $\eta, \phi$ in the second sampling layer of the EM calorimeter (ECAL) was used, together with the electron cluster lateral shower width and number of TRT high threshold hits. A scaled 
isolation variable, defined as the transverse energy within $0.1<\Delta R<0.4$ around the cluster divided by cluster $E_{\mathrm{T}}$, was required below $30 \%$. The fraction of the LJ energy found in the ECAL $\left(f_{E M}\right)>0.98$ was used to reject activity from hadrons.

Single muon-jet (MJ) events were selected from events satisfying a trigger with a single muon having $p_{\mathrm{T}}>18$ $\mathrm{GeV}$. Candidates for double MJs were taken with either a single-muon trigger with $p_{\mathrm{T}}>18 \mathrm{GeV}$ or a three-muon trigger with $p_{\mathrm{T}}>6 \mathrm{GeV}$. Muon candidates must have been reconstructed in both the ID and the MS. Additional requirements were made on the number of associated hits in the silicon pixel and microstrip detectors, as well as on the number of track segments in the MS. The muons were required to come from the PV. The MJs were reconstructed in an iterative procedure using all candidate muons, by seeding the jet candidate with the highest- $p_{\mathrm{T}}$ muon, and adding all muons within $\Delta R<0.1$ : additional jets were formed using the remaining muons. For the double MJ analysis, two muons with $p_{\mathrm{T}}>11 \mathrm{GeV}$ were required per jet with the leading muon $p_{\mathrm{T}}$ be greater than $23 \mathrm{GeV}$ for the single muon trigger events. For the single MJ analysis, four muons were required per jet with $p_{\mathrm{T}}>19,16$, $14 \mathrm{GeV}$, respectively, for the three highest-pT muons, and $p_{\mathrm{T}}>4 \mathrm{GeV}$ for all additional muons. Within a MJ, the two muons closest in $p_{\mathrm{T}}$ were required to have an invariant mass $<2 \mathrm{GeV}$. To suppress muons from hadronic jets, a scaled isolation variable was formed by summing the $E_{\mathrm{T}}$ of all calorimeter cells within $\Delta R=0.3$ of any of the MJ's muons while excluding cells found within $\Delta R=0.05$, and dividing by the $\mathrm{MJ} p_{\mathrm{T}}$. The scaled isolation was required to be less than $0.3(0.15)$ per MJ for the double (single) MJ analyses.

An ABCD method [5] was used to estimate background. For the EJ analysis, $R_{\eta 2}$ and $f_{E M}$ on the second highest$E_{\mathrm{T}} \mathrm{LJ}$ were used to define the four regions: in photon+jet events, the photon will typically deposit more energy in the EM calorimeter than the hadronic jet. The double (single) MJ analysis used the scaled isolation variable and the $p_{\mathrm{T}}$ cut on the fourth (third) muon in the event, associated with a MJ. In the absence of signal, the numbers of events predicted in the signal region for the single MJ channel, the double MJ channel, and the double EJ channel are $3.0 \pm 0.0,0.5 \pm 0.3,15.2 \pm 2.7$ respectively, to be compared with events observed in nearly $5 \mathrm{fb}^{-1}$ of data collected in 20117,3 and 15 for the three analyses. The $95 \%$ confidence level (CL) upper limits on the number of expected events from new phenomena producing collimated pairs of prompt leptons were calculated using the CLs [6] method and reported in Table 1. The observed limits in the EJ channel are in good agreement with the expected limits. The limits in the MJ channels are slightly higher than expected as a result of the slight excesses, but are within $2 \sigma$ of the SM expectation for both channels.

\section{$3 \mathrm{~W}+$ prompt electron jets}

The associated production $\mathrm{pp} \rightarrow \mathrm{WH}$ (assuming SM couplings between the Higgs boson and the $\mathrm{W}$ boson) is stud-

\begin{tabular}{|c|c|c|c|}
\hline $\begin{array}{c}m_{\gamma} \\
(\mathrm{MeV})\end{array}$ & $\begin{array}{c}\text { EJ } \\
\text { Obs (Exp) pb }\end{array}$ & $\begin{array}{c}1 \mathrm{MJ} \\
\text { Obs (Exp) pb }\end{array}$ & $\begin{array}{c}2 \mathrm{MJ} \\
\text { Obs (Exp) pb }\end{array}$ \\
\hline 150 & $0.082(0.082)$ & - & - \\
\hline 300 & $0.11(0.11)$ & $0.060(0.035)$ & $0.017(0.011)$ \\
\hline 500 & $0.20(0.21)$ & $0.15(0.090)$ & $0.019(0.012)$ \\
\hline 150 & $0.096(0.10)$ & 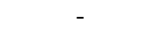 & - \\
\hline 300 & $0.37(0.37)$ & $0.064(0.036)$ & $0.018(0.011)$ \\
\hline 500 & $0.39(0.39)$ & $0.053(0.035)$ & $0.018(0.011)$ \\
\hline 150 & $0.11(0.11)$ & - & - \\
\hline 300 & $0.40(0.40)$ & $0.099(0.055)$ & $0.020(0.012)$ \\
\hline 500 & $1.2(1.2)$ & $0.066(0.043)$ & $0.022(0.015)$ \\
\hline
\end{tabular}

Table 1: Observed and expected limits for the prompt LJ analysis [5].

ied in [7]. The Higgs boson decays, either via a threestep or a two-step cascade to hidden sector particles whose masses are taken to be lower than the Higgs mass (100 $140 \mathrm{GeV}$ ), thus the Higgs decay has a two-LJ topology. The models feature a $\gamma_{d}$ that kinetically mixes with the SM photon, a neutral weakly interacting stable scalar and two hidden scalars. A value of the kinetic mixing parameter $\epsilon>10^{-5}$ implies $\gamma_{d}$ with very short lifetimes; the chosen value of $\epsilon=10^{-4}$ ensures that the decay products are prompt. The $\gamma_{d}$ mass must be less than $2 \mathrm{GeV}$ to provide a viable explanation of the results of cosmic-ray and dark matter direct detection experiments [8], which observe an unexpected excess of cosmic electrons and/or positrons, while there is no observed proton excess. For this analysis, $m_{\gamma}=100$ and $200 \mathrm{MeV}$ are considered, providing decays in $e^{+} e^{-}$pairs only. The signal has a distinct two-jet topology with each EJ having a multiplicity of $>4$ electrons per jet, where the electrons are highly collimated.

The $\mathrm{W}$ boson produced in association with the Higgs boson is reconstructed in the $W \rightarrow e v$ and $W \rightarrow \mu \nu$ decay modes in order to achieve a high efficiency for online event selection and a high signal-to-background ratio. The signal topology is an isolated large $p_{\mathrm{T}}$ lepton accompanied by missing transverse momentum $\left(E_{\mathrm{T}}^{\text {miss }}\right)$ and two or more EJs. For the $W \rightarrow$ ev channel, at least one reconstructed electron trigger object with $p_{\mathrm{T}}>22 \mathrm{GeV}$ is required. For the $W \rightarrow \mu v$ channel, a muon candidate trigger object with $p_{\mathrm{T}}>18 \mathrm{GeV}$, reconstructed in both the ID and MS, is required. The muon trigger object must be consistent with having originated from the interaction region. In both cases, the leptons are required to match the trigger objects in $\Delta R<0.1$.

Signal events are required to have exactly one reconstructed $\mathrm{W}$ boson candidate in the e or $\mu$ decay channel and at least two jets identified as EJs and $E_{\mathrm{T}}^{\mathrm{miss}} \geq 25 \mathrm{GeV}$. The electron from the $\mathrm{W}$ decay is required to pass the tight electron selection $p_{\mathrm{T}}>25 \mathrm{GeV}$, while the $\mu$ candidate is required to be identified in both the inner detector (ID) and the muon spectrometer (MS) subsystems and to have $p_{\mathrm{T}}>20 \mathrm{GeV}$. To increase the robustness against track mis-reconstruction, the difference between the ID and MS $p_{\mathrm{T}}$ measurements is required to be $<15 \mathrm{GeV}$. To reduce background from multi-jet events, electron and muon candidates are required to satisfy an isolation criterion: the sum of $p_{\mathrm{T}}$ of all tracks in a $\Delta R=0.4$ cone around the elec- 
tron (muon) divided by the electron (muon) $p_{\mathrm{T}}$ is required to be less than $0.3(0.2)$. Events with two or more isolated same-flavor leptons are rejected, substantially reducing the background from Drell-Yan production. To reduce the background from cosmic rays, heavy-flavor production and photon conversions, the $\mathrm{W}$ candidate is required to originate from the PV.

The electrons in an EJ are too closely collimated to be identified efficiently with the algorithm used for electrons from $\mathrm{W}$ boson decays and thus are identified with the jet EM fraction $\left(f_{E M}\right) \geq 0.99$ and the jet charged particle fraction $\left(f_{C H}\right) \geq 0.66$, defined as the fraction of jet energy deposited in calorimeter cells that are associated with tracks within the jet. Tracks associated to the jet in a $\Delta R=0.4$ cone $\left(N_{\text {track }}\right)$ must have $p_{\mathrm{T}} \geq 5 \mathrm{GeV}$, total number of pixel and SCT hits $\left(N_{P I X}+N_{S C T}\right)>7$, the fraction of high threshold TRT hits $\left(f_{H T}\right) \geq 0.08$ and be $N_{\text {track }} \geq 2$. Hadronic jets with large $f_{E M}$ are expected to contain mostly neutral pions decaying to photons and, therefore, fewer charged tracks and low $f_{\mathrm{CH}}$. Photons that convert to $e^{+} e^{-}$pairs in the material before entering the calorimeter increase the value of $f_{C H}$.

The dominant background in this search is due to the associated production of a $\mathrm{W}$ boson with hadronic jets which mimic the EJ signature. The background contamination in the signal region is estimated from the data using a simplified matrix method which is completely data-driven, which has been found to be consistent with the data-driven ABCD estimation [7]. Events are assigned to one of the four regions according to whether or not the jets meet the $f_{E M}$ and the track-quality conditions. In the signal region, two jets with $f_{E M} \geq 0.99$ are required and for both jets $N_{\text {track }}$ has to be $>2$. The estimated background is $0.41 \pm 0.29 \pm 0.12$ events, while only one event has been observed in $2.04 \mathrm{fb}^{-1}$ of 2011 data in the $W \rightarrow \mu \nu$ channel. The expected signal yields are reported in Table 2. Assuming SM cross section for the WH production, Higgs branching ratios to EJs between 24 and $45 \%$ are excluded at $95 \%$ CL (Figure 1).

\section{Displaced muon-jet search}

The benchmark model for this analysis [9] is a simplified scenario where the Higgs boson decays to a pair of neutral hidden fermions $\left(f_{d 2}\right)$ each decaying to one long-lived $\gamma_{d}$ and one stable neutral hidden fermion $\left(f_{d 1}\right)$ that escapes

\begin{tabular}{c|c|c}
$\mathrm{m}_{H}(\mathrm{GeV})$ & $m_{\gamma}=100 \mathrm{MeV}$ & $m_{\gamma}=200 \mathrm{MeV}$ \\
\hline & 2-step model & \\
\hline 100 & $14.3 \pm 1.7 \pm 0.8$ & $12.4 \pm 1.6 \pm 0.7$ \\
125 & $11.3 \pm 1.0 \pm 0.6$ & $10.7 \pm 1.1 \pm 0.6$ \\
140 & $9.6 \pm 0.8 \pm 0.5$ & $9.0 \pm 0.8 \pm 0.4$ \\
\hline & 3 -step model & \\
\hline 100 & $22.6 \pm 2.1 \pm 1.2$ & $23.5 \pm 2.1 \pm 1.2$ \\
125 & $16.2 \pm 1.2 \pm 0.9$ & $18.1 \pm 1.4 \pm 1.0$ \\
140 & $13.7 \pm 0.9 \pm 0.8$ & $13.9 \pm 0.9 \pm 0.8$
\end{tabular}

Table 2: Expected events in signal MC for $\mathrm{W}+$ prompt $\mathrm{LJ}$ analysis [7]. the detector unnoticed, resulting in two MJs from the $\gamma_{d}$ decays in the final state. Parameters for MC simulations are given in Table 3. Since signal events are characterized by a four-muon final state with relatively low $p_{\mathrm{T}}$, a low $p_{\mathrm{T}}$ multi-muon trigger with muons reconstructed only in the MS is needed. In order to have an acceptably low trigger rate, at least three muons are required. Candidate events are collected using an unprescaled high level trigger with three reconstructed muons of $p_{\mathrm{T}} \geq 6 \mathrm{GeV}$, seeded by a Level 1 trigger with three different muon RoIs. These muons are reconstructed using the tracking at the trigger level only in the MS, since muons originating from a neutral particle decaying outside the pixel detector will not have a matching track in the ID. The trigger efficiency for the MC signal samples, defined as the fraction of events passing the trigger requirement with respect to the events satisfying the analysis selection criteria is $0.32 \pm 0.01_{\text {stat }}$ for $m_{H}=100 \mathrm{GeV}$ and $0.31 \pm 0.01_{\text {stat }}$ for $m_{H}=140 \mathrm{GeV}$. The main reason for the relatively low trigger efficiency is the small opening angle $(\Delta R)$ between the two muons of the $\gamma_{d}$ decay, which is often smaller than the Level 1 trigger granularity.

MJs are identified by a clustering algorithm that associates all the muons in $\Delta \mathrm{R}=0.2$ cones, starting with the muon with highest $p_{\mathrm{T}}$. The MJ direction and momentum are obtained from the vector sum over all muons in the MJ. Only events with two MJs separated with $|\Delta \phi| \geq 2$ and each containing two reconstructed muons of opposite charge, are kept for the analysis. The main background contribution is expected from processes giving a high production rate of secondary muons which do not point to the primary vertex, such as decays in flight of $\mathrm{K} / \pi$ and heavy flavour decays in multi-jet processes, or muons due to cosmic rays.

The calorimetric isolation variable $E_{\mathrm{T}}^{\text {isol }}$ has been defined as the difference between the $E_{\mathrm{T}}$ in a $\Delta \mathrm{R}=0.4$ cone around the highest $p_{\mathrm{T}}$ muon and the $E_{\mathrm{T}}$ in a 0.2 cone; a cut $E_{\mathrm{T}}^{\text {isol }}$ $\leq 5 \mathrm{GeV}$ keeps almost all the signal and significantly reduce the background. The isolation modelling is validated

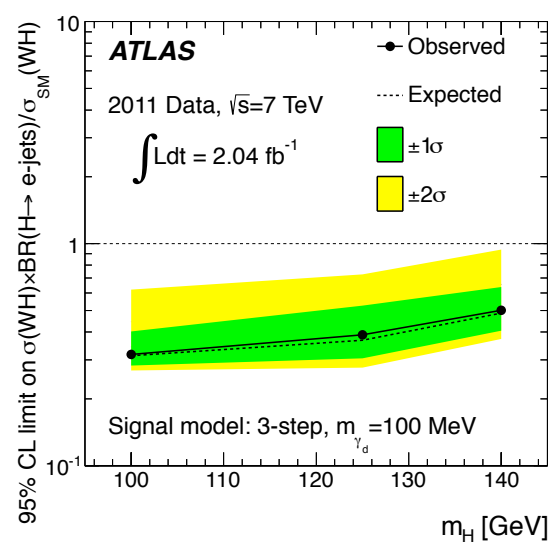

Figure 1: Observed and expected 95\% CL upper limits on the $\sigma(W H) \times B R(H \rightarrow E J S) / \sigma_{S M}(W H)$ as function of the Higgs mass for the 3-step model for the $\mathrm{W}+$ prompt $\mathrm{LJ}$ analysis [7]. 


\begin{tabular}{ccccc}
$\begin{array}{c}m_{H} \\
(\mathrm{GeV})\end{array}$ & $\begin{array}{c}m_{f d 1} \\
(\mathrm{GeV})\end{array}$ & $\begin{array}{c}m_{f d 2} \\
(\mathrm{GeV})\end{array}$ & $\begin{array}{c}m_{\gamma d} \\
(\mathrm{GeV})\end{array}$ & $\begin{array}{c}c \tau \\
(\mathrm{mm})\end{array}$ \\
\hline 100 & 5.0 & 2.0 & 0.4 & 47 \\
\hline 140 & 5.0 & 2.0 & 0.4 & 36 \\
\hline
\end{tabular}

Table 3: MC simulation for the displaced MJ analysis [9].

with a sample of $Z \rightarrow \mu \mu$ decays. The scalar sum of the $\mathrm{p}_{T}$ of the tracks measured in the ID $\left(\Sigma_{p_{\mathrm{T}}}^{\mathrm{ID}}\right)$, inside a $\Delta \mathrm{R}=0.4$ cone around the direction of the MJ, is requested to be $<3 \mathrm{GeV}$. The muon tracks of the MJ in the ID, if any, are not removed from the isolation sum; as a consequence, the $\Sigma_{p_{\mathrm{T}}}^{\mathrm{ID}}$ cut will remove MJs with very short decay length.

For the cosmic-ray muon background, we require the transverse and longitudinal impact parameters of the muons with respect to the primary vertex to be $\left|\mathrm{d}_{0}\right|<200 \mathrm{~mm}$ and $\left|\mathrm{z}_{0}\right|<270 \mathrm{~mm}$. The $\gamma_{d}$ reconstruction efficiency for the lifetimes used in this simulation, defined as the number of $\gamma_{d}$ passing the offline selection divided by the number of $\gamma_{d}$ in the MS acceptance $(|\eta|<2.4)$ with both muons having $p_{\mathrm{T}} \geq 6 \mathrm{GeV}$, is around $35 \%$. To estimate the multi-jet background contamination in the signal region we use a data-driven $\mathrm{ABCD}$ method [9], slightly modified in order to cope with the low statistics: the two relatively uncorrelated variables used to separate signal and background are the $\mathrm{MJ} E_{\mathrm{T}}^{\mathrm{isol}}$ and $\Delta \phi$. No events in the data sample pass the selection with an expected total background of $0.06 \pm 0.02_{\text {stat }}^{+0.66}{ }_{-0.6}$ syst events.

The efficiency of the selection criteria is evaluated for the simulated signal samples as a function of the mean lifetime of the $\gamma_{d}$, so the expected number of signal events is predicted in a $c \tau$ up to $700 \mathrm{~mm}$. These numbers, together with the expected number of multi-jet and cosmic ray events, no data events surviving the selection criteria in $1.9 \mathrm{fb}^{-1}$ and all the systematic uncertainties, are used as input to obtain limits at the $95 \% \mathrm{CL}$ on the $\sigma \times \mathrm{BR}$ for the process $H \rightarrow \gamma_{d} \gamma_{d}+\mathrm{X}$ through the CLs method (Figure 2).

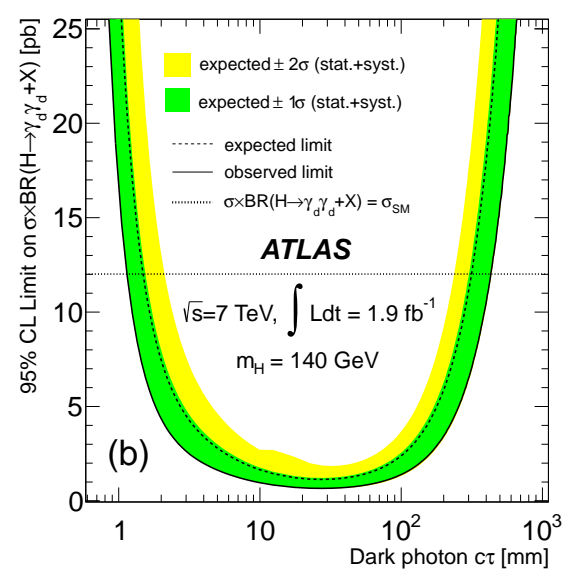

Figure 2: 95\% upper limits on the $\sigma \times B R$ as a function of $\gamma_{d} c \tau$ for the displaced MJ analysis for Higgs boson mass $=140 \mathrm{GeV}[9]$.

\section{Displaced $\pi_{v}$ decays in the MS}

This analysis describes the first ATLAS search for the Higgs decay to two identical neutral particles $\left(\pi_{v}\right)$ that have displaced decay $b \bar{b}, c \bar{c}, \tau^{+} \tau^{-}$in the ratio 85:5:8 [10]. Four datasets have been simulated with Higgs masses 120 and $140 \mathrm{GeV}$ and $\pi_{v}$ masses 20 and $40 \mathrm{GeV}$. Both $\pi_{v}$ decays are required to occur near the outer radius of the hadronic calorimeter (HCAL) ( 4 m) or in the MS. Such decays give a $(\eta, \phi)$ cluster of charged and neutral hadrons in the MS. Requiring both $\pi_{v}^{\prime} s$ to have this topology improves background rejection. A dedicated signature-driven trigger, the muon Region Of Interests (RoI) cluster trigger [11], was developed to trigger on events with a $\pi_{v}$ decaying in the MS. It selects events with a cluster of three or more muon RoIs in a $\Delta \mathrm{R}=0.4$ cone in the MS barrel trigger chambers: one $\pi_{v}$ must decay in the barrel, while the second $\pi_{v}$ may decay also in the forward MS.

Punch-through jets are suppressed by requiring no calorimeter jets with $E_{\mathrm{T}}>30 \mathrm{GeV}$ in a $\Delta \mathrm{R}=0.7$ cone and no ID tracks with $p_{\mathrm{T}}>5 \mathrm{GeV}$ within a region of $\Delta \eta \times \Delta \phi=0.2 \times 0.2$ around the RoI cluster center. Monte Carlo (MC) studies show the RoI cluster trigger is 30-50\% efficient in the region from $4 \mathrm{~m}$ to $7 \mathrm{~m}$. The $\pi_{v}^{\prime} s$ that decay beyond $7 \mathrm{~m}$ do not leave hits in the trigger chambers located at $7 \mathrm{~m}$, while the $\pi_{v}$ decays that occur before $4 \mathrm{~m}$ are located in the calorimeter and do not produce sufficient activity in the MS. A specialized tracking and vertex reconstruction algorithm was developed to identify $\pi_{v}^{\prime} s$ that decay inside the MS. Such decays produce a high multiplicity of low $p_{\mathrm{T}}$ particles clustered in a small $\Delta \mathrm{R}$ region, containing $\sim 10$ charged particles and $\sim 5 \pi_{0}$, resulting in large EM showers, which confuses standard muon reconstruction. The $\pi_{v}^{\prime} s$ that decay before the last sampling layer of the HCAL do not produce a significant number of tracks in the MS. Thus, detectable decay vertices must be located in the region between the outer radius of the HCAL and the middle station of the MS. The separation of the two multilayers inside a single muon chamber provides a powerful tool for track pattern recognition in this busy environment and a momentum measurement with resolution for tracks up to $\sim 10 \mathrm{GeV}$ in the barrel. The MS vertex algorithm begins by grouping the track segments formed out of hits in single muon chambers using a simple cone algorithm with $\Delta \mathrm{R}=0.6$. In the barrel the vertex is reconstructed as the point in $(r, z)$ that uses the largest number of track segments to reconstruct a vertex with a $\chi^{2}$ probability greater than $5 \%$, while in the forward MS, the vertex is found using a least squares regression, that assumes the track segments are straight lines.

A vertex is reconstructed using at least three track segments. After requiring the vertex to be separated from ID tracks with $p_{\mathrm{T}} \geq 5 \mathrm{GeV}$ and jets with $E_{\mathrm{T}} \geq 15 \mathrm{GeV}$ by $\Delta \mathrm{R}=0.4$ and 0.7 , respectively, the algorithm has an efficiency of $\sim 40 \%$ in signal MC events throughout the barrel region (4 $r \leq 7.5 \mathrm{~m}$ ) (Figure 3 (left)) and a resolution of $20 \mathrm{~cm}$ in $z, 32 \mathrm{~cm}$ in $r$ and $50 \mathrm{mrad}$ in $\phi$. In the forward MS, the algorithm is $40 \%$ efficient in the region $8 \leq|z| \leq 14 \mathrm{~m}$. The MC description of hadrons and photons in the MS was validated on a sample of events 

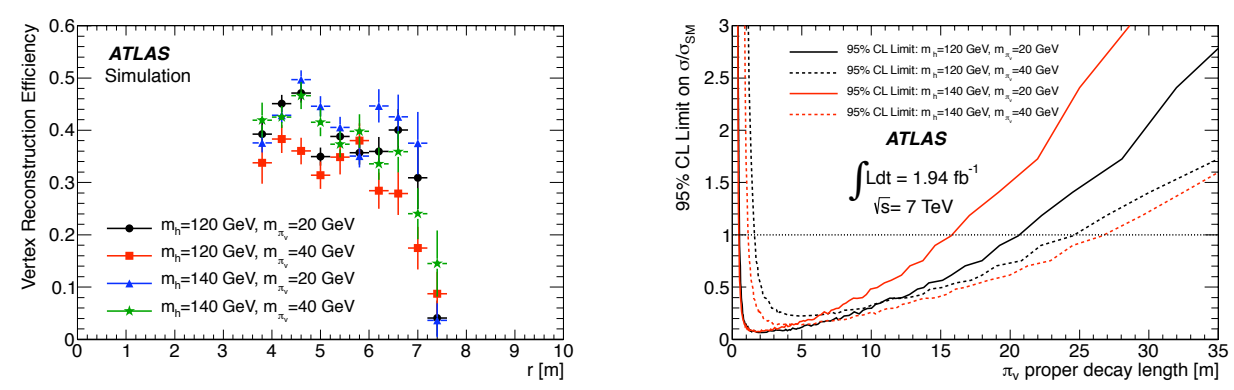

Figure 3: Vertex reconstruction efficiency as a function of the radial decay position of the $\pi_{v}$ and observed $95 \%$ upper limits on $H \rightarrow \pi_{v} \pi_{v}$ as a function of the $\pi_{v}$ proper decay length for the displaced $\pi_{v}$ search [10].

containing a punch-through jet, which are similar to signal events as they contain both low energy photons and charged hadrons in a localized region of the MS. The final event selection requires two isolated MS vertices separated by $\Delta R>2$. The background of $0.03 \pm 0.02$ events is calculated using a fully data-driven method by measuring the probability for a random event to contain an MS vertex $\left(\mathrm{P}_{\text {vertex }}\right)$ and the probability of reconstructing a vertex given the event passed the trigger $\left(\mathrm{P}_{\text {reco }}\right)$. $\mathrm{P}_{\text {vertex }}$ was measured using events selected by a random generator in coincidence with the bunch crossing and $\mathrm{P}_{\text {reco }}$ was measured on collision data from events that pass the trigger. No events in the data have two isolated, back-to-back vertices in the MS. Since no significant excess over the background prediction is found, exclusion limits for $\sigma_{H} \times B R\left(H \rightarrow \pi_{v}\right.$ $\pi_{v}$ ) as a function of the $\pi_{v}$ proper decay length $(c \tau)$ are set by rejecting the signal hypothesis at the $95 \% \mathrm{CL}$, as shown in Figure 3 (right).

\section{Conclusions}

In 2011 pp collisions at $7 \mathrm{TeV}$ recorded with the ATLAS detector at the LHC, there is no evidence of an excess of events for any of the analyses described in this paper. A search for collimated pairs of leptons has been performed on nearly $5 \mathrm{fb}^{-1}$ : no significant excess of data compared to the SM expectation was observed in any of the three channels, and 95\% CL upper limits have been set on the $\sigma \times \mathrm{BR}$ for several parameters of a Hidden Valley model. A search is also presented for a light Higgs boson decaying to highly collimated jets of electrons, which are expected to be seen in the detector as distinct prompt electron-jets. The analysis has been performed using $2.04 \mathrm{fb}^{-1}$ in the WH production mode. The observed data are consistent with the SM background hypothesis. Limits at 95\% CL are set on the WH production $\sigma \times \mathrm{BR}$ into electron-jets. No excess has been observed for the process $H \rightarrow \gamma_{d} \gamma_{d}+\mathrm{X}$ : assuming the SM production rate for a $140 \mathrm{GeV}$ Higgs, its BR to two $\gamma_{d}^{\prime} s$ is found to be below $10 \%$ in the $c \tau$ range from 7 to $82 \mathrm{~mm}$. For events with two isolated back-toback vertices in the ATLAS MS, a wide range of $\pi_{v}$ proper decay length from 0.5 to $25 \mathrm{~m}$ can be excluded, assuming $100 \%$ BR for $H \rightarrow \pi_{v} \pi_{v}$.

\section{References}

[1] ATLAS Collaboration, Phys. Lett. B 716 1-29 (2012)

[2] CMS Collaboration, Phys. Lett. B 716 30-61 (2012)

[3] M. J. Strassler, Phys. Lett. B 661, 263-267 (2008)

[4] ATLAS Collaboration, JINST 3 S08003 (2008)

[5] ATLAS Collaboration, Phys. Lett. B 719, 299 (2013)

[6] A. Read, J. Phys. G 28, 2693 (2002)

[7] ATLAS Collaboration, NJP 15 (2013) 043009

[8] HEAT Collaboration, Astr. J. Lett. 482 L191 (1997)

Fermi LAT Collaboration, PRL 102181101 (2009)

PAMELA Collaboration, Nature 458 607-9

Chang J et al, Nature 456 362-5

[9] ATLAS Collaboration, arXiv:1210.0435

[10] ATLAS Collaboration, PRL 108251801 (2012)

[11] ATLAS Collaboration, ATL-PHYS-PUB-2009-082

[12] A. Falkowski et al., JHEP 05 (2010) 077

A. Falkowski et al., PRL 105241801 (2010) 